\title{
Differences in Access to Outpatient Care in the State of Ohio for an Orthopaedic Sports Medicine Patient
}

\author{
Robert N. Matar, M.D., Colin D. F. Cotton, B.A., Violet T. Schramm, Nihar S. Shah, B.S., and \\ Brian M. Grawe, M.D.
}

\begin{abstract}
Purpose: To evaluate the impact of either Medicaid or private insurance on securing an appointment in an outpatient orthopaedic clinic and to determine waiting periods until an appointment as well as the relationship between population metrics and access to care. Methods: A total of 88 clinics were called. There were 2 fictitious patients, one with an anterior cruciate ligament (ACL) injury and the other with a medial meniscus injury, with each calling as having Medicaid or private insurance. Clinic responses were recorded for whether an appointment could be made, when it was scheduled, and with what provider. Results: A total of 32 of 88 (36.4\%) of the clinics scheduled an appointment for the Medicaid patient reporting an ACL injury versus 71 of $88(80.6 \%)$ of the clinics that scheduled an appointment for the same patient with private insurance. A total of 34 of $88(38.6 \%)$ of the clinics scheduled an appointment for the Medicaid patient reporting a medial meniscus injury versus 71 of $88(80.6 \%)$ of the clinics that scheduled an appointment for the same patient with private insurance. Mean waiting period for ACL patients with Medicaid was 8.6 days and 4.5 days for patients with private insurance, whereas medial meniscus patients with Medicaid was 7.7 days and 5.4 days for patients with private insurance. In total, 60 of the $66(90.9 \%)$ patients with Medicaid who received an appointment were scheduled to see the orthopaedic surgeon (30 in both ACL and medial meniscus groups). In total, 126 (71.6\%) patients with Medicaid and $34(19.3 \%)$ patients with private insurance of the 176 encounters faced barriers to scheduling an appointment. Rural communities were associated with appointment acceptance for patients with Medicaid $(P<.05)$, and patients with private insurance had successful appointment scheduling in all community types $(P<.05)$. Conclusions: This study suggests that patients with Medicaid are less likely to receive orthopaedic care for multiple sports medicine injuries, are more likely to encounter barriers, and endure longer waiting periods. There are different patterns of insurance acceptance according to population metrics. Clinical Relevance: Serves as a baseline evaluation of the difference in access to health care that may be impacted by increases in Medicaid coverage and/or changes in government policies.
\end{abstract}

\section{Introduction}

$\mathbf{M}$ edicaid is a partnership between state and federal programs that, in conjunction with the Children's Health Insurance Program, affords more than 70 million Americans with health coverage. ${ }^{1}$ It is the largest provider of health insurance in the United

From the Department of Orthopaedics and Sports Medicine, University of Cincinnati, Cincinnati, Ohio, U.S.A.

The authors report that they have no conflicts of interest in the authorship and publication of this article. Full ICMJE author disclosure forms are available for this article online, as supplementary material.

Received March 3, 2020; accepted July 18, 2020.

Address correspondence to Nihar S. Shah, B.S., Department of Orthopaedics and Sports Medicine, 231 Albert Sabin Way, Cincinnati, OH 452670212.E-mail:Nss86@case.edu

(C) 2020 THE AUTHORS. Published by Elsevier Inc. on behalf of the Arthroscopy Association of North America. This is an open access article under the CC BY-NC-ND license (http://creativecommons.org/licenses/by-nc-nd/4.0/). 2666-061X/20226

https://doi.org/10.1016/j.asmr.2020.07.015
States. $^{2}$ Beneficiaries include children, pregnant women, senior citizens, and disabled individuals. ${ }^{3}$

Progress was made when the Patient Protection and Affordable Care Act (PPACA) of 2010 was passed, which provided the opportunity for states to broaden the eligibility for nearly all Americans younger than the age of 65 years. It means that all adults with incomes at or below $133 \%$ the poverty level, (i.e., $<\$ 34,248$ for a family of 4) can become eligible under the PPACA. ${ }^{4}$

With recent changes to the health insurance environment, there may be differences in access to care for orthopaedic patients. Current literature supports early repair of anterior cruciate ligament (ACL) ruptures and meniscal tears. ${ }^{5-8}$ To not only allow timely access to orthopaedic care for the adult sports medicine population but also ensure that patients are receiving the proper treatment, barriers to treatment must be identified.

There is limited literature evaluating orthopaedic sports medicine injuries and insurance. One such study 
by Wiznia et al. ${ }^{9}$ used a bucket-handle meniscus tear. The authors found that $91.2 \%$ of offices had scheduled an appointment for the patient with private insurance but only $27.1 \%$ for the patient with Medicaid $(P<.0001)$. Furthermore, patients with Medicaid waited longer for appointments (15 days) versus patients with private insurance $(12$ days $)(P<.0001)$.

Similarly, a study performed by Patterson et al. ${ }^{10}$ evaluated rotator cuff injuries. The group discovered that $96 \%$ of offices had scheduled an appointment for the patient with private insurance but only $72 \%$ for the patient with Medicaid $(P<0.001)$. The present study evaluates the effect of population metrics on waiting period to appointment, the differences in the type of the provider to be seen, and the reasons for appointment denial. In addition, this study will allow clinicians to take notes in how practice patterns are changing with evolving health care policy when compared with previous studies.

The purpose of this study was to evaluate the impact of either Medicaid or private insurance on securing an appointment in an outpatient orthopaedic clinic and to determine waiting periods until an appointment as well as the relationship between population metrics and access to care. We hypothesized that patients reporting as having Medicaid insurance would be less likely to secure an appointment, have longer waiting periods until scheduled appointments, and have different patterns of acceptance according to population metrics when compared with patients with private insurance.

\section{Methods}

All members of the Ohio Orthopaedic Society (OOS) and American Orthopaedic Society for Sports Medicine (AOSSM) with an Ohio location were identified on each society's respective website. Ninety unique clinics were identified via OOS directories and AOSSM directories. An additional 11 were added via searches performed on yellowpages.com, using the terms "orthopaedic surgeon," "orthopaedic clinic," "orthopedic surgeon," and "orthopedic clinic," that were not already identified by OOS and AOSSM directories. Clinics with multiple locations were only counted and used once. This left a total of 101 clinics.

Each of the clinics were called. If a number was disconnected or inactive, it was excluded. Calls were made over a 3-month period and provided the following story: "I injured my knee while playing soccer a few days ago. I was initially treated in an emergency room and had both an X-ray and magnetic resonance imaging performed. I was told that I have a torn ACL and will likely need surgery. When is your first available office appointment with a surgeon who could evaluate me?" The same script was used for the patient calling with a torn medial meniscus, except medial meniscus was substituted into the description. These calls were made, on average, 1 week apart. Calls were made by 2 different authors (C.C. and V.S.) to ensure the caller was not recognized. For the first call, in each injury group, the clinic was told that the fictitious patient had an Ohio-based Medicaid insurance plan (i.e., CareSource). For the second call, the clinic was told that the fictitious patient had an Ohio-based private insurance plan (i.e., Anthem Blue Cross Blue Shield). Clinic responses were recorded for whether an appointment could be made, when it was scheduled, and with what provider (e.g., physician, physician assistant). If the fictitious patient was not able to schedule an appointment, a reason for why was recorded, and a referral name also was recorded. Any barriers for the fictitious patient to be seen also were recorded. Some examples include requiring federal ID numbers or primary care physician referrals. Any circumstance in which a barrier was encountered, the call was considered as being unable to secure an appointment. Clinics also were evaluated based on population and demographic metrics, such as the type of clinic (e.g., private or academic), community type (e.g., urban, suburban, rural), and population density. Practices were considered academic if affiliated with a university, community if part of a hospital independent of a university, and private if wholly owned by the physicians.

\section{Statistical Analysis}

According to a previously described methodology, a minimum of 88 clinics are needed to be powered enough to detect a Cohen effect size of at least 0.2 between Medicaid and private insurance acceptance rates. ${ }^{9,11}$ This value provides a benchmark, that if the standard deviation between the Medicaid and private insurance groups are not greater than 0.2, then the difference between the 2 insurance groups is inconsequential, even if the values are statistically significant. $\chi^{2}$ tests were used to evaluate the difference in rates of securing appointments and reasons for denial between insurance types. Fisher exact tests were used if greater than $20 \%$ of the expected values were less than 5 . Furthermore, linear regression analysis was carried out to evaluate whether population density could be a prognosticator for determining the appointment waiting period. Unless otherwise noted, 2 -tailed $t$ tests were performed for association of continuous variables.

\section{Results}

In total, 88 clinics were called between July and August of 2019. In total, 352 calls were made for both ACL and medial meniscus groups as either a Medicaid or private insurance patient. In total, 32 of 88 (36.8\%) of the clinics scheduled an appointment for the patient with Medicaid reporting an ACL injury versus 71 of 88 $(81.6 \%)$ of the clinics that scheduled an appointment for the same patient with private insurance. In total, 34 
of $88(38.6 \%)$ of the clinics scheduled an appointment for the patient with Medicaid reporting a medial meniscus injury versus 71 of $88(80.6 \%)$ of the clinics that scheduled an appointment for the same patient with private insurance (Table 1).

After the rate of receiving an appointment was recorded, the type of provider that would be evaluating the fictitious patient was documented. In total, 60 of the 66 Medicaid patients who received an appointment $(90.9 \%)$ were scheduled to see the orthopaedic surgeon (30 in both ACL and medial meniscus groups), zero were scheduled to see a primary care specialist, and 6 of the $66(9.1 \%)$ were scheduled to see a physician assistant or nurse practitioner ( 2 and 4 for ACL and medial meniscus groups, respectively) (Table 1). There was no statistically significant association between insurance type and the provider to be seen $(P=.33)$.

The patients were then evaluated for the waiting period from the time of the call to the time of the scheduled appointment. Mean waiting period for ACL patients with Medicaid was 8.6 days and 4.5 days for patients with private insurance. Mean waiting period for medial meniscus patients with Medicaid was 7.7 days and 5.4 days for patients with private insurance (Table 2).

There were a multitude of barriers encountered by patients. Overall, of the 176 Medicaid patient encounters, $126(71.6 \%)$ reported barriers to scheduling an appointment. Only $38(21.6 \%)$ were due to the insurance type, whereas $61(48.4 \%)$ were attributed to requiring additional referral and imaging data. In contrast, only 34 of the $176(19.3 \%)$ private insurance patient encounters reported barriers to scheduling an appointment. $\chi^{2}$ analysis demonstrates that there was a statistically significant difference in the number of barriers encountered by the patients with Medicaid compared to the patients with private insurance $(P<$ .05). Data can be found in Table 3.

After the clinic supplied the reason for not securing an appointment, clinics were asked to provide the name of an alternate provider nearby. Only those patients calling as a patient with Medicaid received answers. Overall, 52 of the 110 denied patients with Medicaid were provided information $(47.2 \% ; 176$ total calls for the combined ACL and medial meniscus group). The guidance included calling the insurance company $(40.4 \%)$, providing a specific name $(25.0 \%)$, visiting a local university $(13.5 \%)$, administrator admitting to being unsure $(15.4 \%)$, calling a primary care physician $(3.80 \%)$, or visiting the emergency department/urgent care $(1.90 \%)$.

In addition to evaluation of appointment acceptance rate, waiting period, and barriers to scheduling appointments, we examined population densities and type of clinical practice. A total of 29 of $88(33.0 \%)$ clinics were in rural settings, 47 of $88(53.4 \%)$ were suburban, and 12 of 88 (13.6\%) were urban. A total of 38 of $88(43.2 \%)$ clinics were private practice, 8 of 88 $(9.1 \%)$ were academic practices, and 42 of $88(47.7 \%)$ were community practices.

Statistical analyses to determine the relationship between insurance type, community locations, time to appointment, and granted appointment rate were performed by combining the ACL and meniscus injury groups for both patient populations. The association between community type and successful appointment scheduling was calculated with $\chi^{2}$ analysis. There was a significant relationship $(P<.05)$. between rural communities and Medicaid appointment acceptance. In addition, patients with private insurance were found to have an association between successful appointment scheduling in all community types $(P<.05)$.

A univariate linear regression model was performed to assess the correlation between population density and both the waiting period until an appointment for patients with Medicaid and patients with private insurance. Neither the patients with Medicaid $(\mathrm{r}=0.12$, $P=.33)$ or the patients with private insurance $(\mathrm{r}=$ $0.02, P=.79$ ) had statistically significant associations between increasing population density and waiting periods until an appointment.

Finally, we evaluated the association between practice type and successful appointment scheduling. Patients with Medicaid had a significantly $(P<.05)$ greater rate of appointment scheduling with community practices. Patients with private insurance had

Table 1. Clinic Responses and Provider to Be Seen

\begin{tabular}{|c|c|c|c|c|}
\hline & \multicolumn{2}{|r|}{ Medicaid } & \multicolumn{2}{|r|}{ Private } \\
\hline Number of clinics offering appointment & $32(36.8 \%)$ & $34(38.6 \%)$ & $71(81.6 \%)$ & $71(81.6 \%)$ \\
\hline Orthopaedic surgeon & $30(93.8 \%)$ & $30(88.2 \%)$ & $67(94.4 \%)$ & $66(93.0 \%)$ \\
\hline Primary care physician & $0(0 \%)$ & $0(0 \%)$ & $1(1.4 \%)$ & $1(1.4 \%)$ \\
\hline Physician assistant or nurse practitioner & $2(6.25 \%)$ & $4(11.8 \%)$ & $3(4.2 \%)$ & $4(5.6 \%)$ \\
\hline
\end{tabular}

NOTE. Success rates and the distribution of provider assigned for orthopaedic sports medicine patients attempting to secure an appointment based on insurance type and diagnosis.

ACL, anterior cruciate ligament. 
Table 2. Latency Period Until Appointment

\begin{tabular}{|c|c|c|c|}
\hline \multicolumn{2}{|c|}{ Medicaid } & \multicolumn{2}{|r|}{ Private } \\
\hline ACL & Medial Meniscus & ACL & Medial Meniscus \\
\hline 8.6 & 7.7 & 4.5 & 5.4 \\
\hline
\end{tabular}

greater rates of appointment acceptance in all types of clinics $(P<.05)$.

\section{Discussion}

This study found that patients with Medicaid have barriers in obtaining an outpatient appointment. Specifically, for both the ACL and medial meniscus groups, patients with Medicaid had more difficulty securing an appointment compared with identical patients with private insurance $(37.5 \%$ vs $81.6 \%$, respectively), encountered more barriers $(71.5 \%$ vs $19.3 \%$, respectively), and endured longer waiting periods until they could be seen in the clinic (8.14 vs 4.95 days).

PPACA has played a role in expanding Medicaid coverage for millions of Americans. ${ }^{12,13}$ Although the number of patients covered by insurance has increased, their access to care may not be the same as compared with those with private insurance. In this study, we evaluated the impact type of insurance may have on securing an appointment in an outpatient orthopaedic clinic. In addition, we evaluated the relationship between population density and access to care.

Past studies on difference in access to care for sports medicine injuries are limited but have shown that there is reduced access to care for patients with Medicaid. ${ }^{9,10}$ This study evaluates orthopaedic sports medicine injuries in a Medicaid expanded state and correlates population metrics with access to care. In addition, this study was performed in late 2019, allowing for analysis of how changes in health care policy has affected access to sports medicine when compared with a previous study.

Patients with Medicaid encountered more barriers $(P<.05)$, such as requiring additional referral and imaging data. This finding suggests that patients with Medicaid may be subject to additional barriers outside of the actual insurance policy coverage. However, if referral and imaging data were true barriers, then individuals presenting with private insurance would be subject to the same barriers, suggesting the true barrier is insurance type and not referral or imaging data. Later, when administrators were asked to provide information for another provider after denial, only $25 \%$ provided a specific name, indicating many patients with Medicaid may continue to have challenges after attempting to schedule their initial appointment. The reason for these barriers requires further study.

When the association between community type and appointment acceptance was evaluated, an association was discovered between rural communities and appointment acceptance for patients with Medicaid $(P<.05)$, and an association between all community types and appointment acceptance for private insurance patients $(P<.05)$. This suggests that patients with Medicaid may find it easier to be evaluated in a rural community, whereas private insurance patients may be evaluated in all community types. Similarly, the data demonstrate that patients with Medicaid are more likely to receive appointments from community practices $(P<.05)$ whereas patients with private insurance can find success at any practice type $(P<.05)$. When using quantitative population metrics, we found a weak positive correlation $(P=.33)$ and no correlation $(P=.79)$ between population and waiting period for Medicaid and private insurance patients, respectively. These data illustrate that the population may not serve as a predictor for wait time until an appointment. Furthermore, wait times between densely populated areas may not be more than that at less-populated rural areas.

\section{Limitations}

The first limitation was inherent in the data-collection aspect of the study. Despite having a prepared script, many administrators asked questions and had unique appointment scheduling procedures leading to heterogeneity in fictitious patient presentation. For this reason, while the order in which the data were presented varied, we ensured that every provider had the same set of information for the scenario.

Although a previous analysis suggested that calling 88 practices would provide enough power to the study, it is still a small sample size and may not be generalizable to all orthopaedic clinics across the nation. Finally, results may have been confounded by logistics and practice specific policies. Mainly, additional information requested that we could not create may have

Table 3. Reasons for Denial

\begin{tabular}{lcc}
\hline & $\begin{array}{c}\text { Medicaid* } \\
(\mathrm{n}=126)\end{array}$ & $\begin{array}{c}\text { Private* } \\
(\mathrm{n}=34)\end{array}$ \\
\hline State or federal ID information needed & 4 & 6 \\
More insurance information needed & 20 & 8 \\
Referral needed & 37 & 12 \\
Insurance not accepted & 38 & 0 \\
Other & 23 & 6 \\
Multiple reasons & 4 & 2 \\
\hline NOTE. Justifications for appointment denial. Both anterior cruciate \\
ligament and medial meniscus groups included in each insurance \\
type. \\
$\quad$ *Both anterior cruciate ligament and medial meniscus groups \\
included in each insurance type.
\end{tabular}


prevented the fictitious patient from securing an appointment.

\section{Conclusions}

This study suggests that patients with Medicaid are less likely to receive orthopaedic care for multiple sports medicine injuries, are more likely to encounter barriers, and endure longer waiting periods. There are different patterns of insurance acceptance according to population metrics.

\section{References}

1. Medicaid \& CHIP: December 2015 Monthly Applications, Eligibility Determinations and Enrollment Report; 2016, http://www.cms.gov/mmrr/Downloads/MMRR2013_ 003_04_a02.pdf. Accessed September 3, 2019.

2. Iglehart JK, Sommers BD. Medicaid at 50-From welfare program to nation's largest health insurer. $N$ Engl J Med 2015;372:2152-2159.

3. Cheung PT, Wiler JL, Lowe RA, Ginde AA. National study of barriers to timely primary care and emergency department utilization among medicaid beneficiaries. Ann Emerg Med 2012;60:4-10.e2.

4. Majerol M, Newkirk V, Garfield R, Kaiser Family Foundation. The uninsured: A primer. Available at: http://files. kff.org/attachment/primer-the-uninsured-a-primer-key-f acts-about-health-insurance-and-the-uninsured-in-theera-of-health-reform. Accessed July 17, 2020.

5. Sommerfeldt M, Goodine T, Raheem A, Whittaker J, Otto D. Relationship between time to ACL reconstruction and presence of adverse changes in the knee at the time of reconstruction. Orthop J Sport Med 2018;6: 232596711881391.
6. Everhart JS, Kirven JC, Abouljoud MM, DiBartola AC, Kaeding CC, Flanigan DC. Effect of delayed primary anterior cruciate ligament reconstruction on medial compartment cartilage and meniscal health. Am J Sports Med 2019;47:1816-1824.

7. Venkatachalam S, Godsiff SP, Harding ML. Review of the clinical results of arthroscopic meniscal repair. Knee 2001;8:129-133.

8. Beaufils P, Becker R, Kopf S, Matthieu O, Pujol N. The knee meniscus: management of traumatic tears and degenerative lesions. EFORT Open Rev 2017;2:195.

9. Wiznia DH, Nwachuku E, Roth A, et al. The influence of medical insurance on patient access to orthopaedic surgery sports medicine appointments under the Affordable Care Act. Orthop J Sport Med 2017;5: 2325967117714140.

10. Patterson BM, Spang JT, Draeger RW, Olsson EC, Creighton RA, Kamath GV. Access to outpatient care for adult rotator cuff patients with private insurance versus Medicaid in North Carolina. J Shoulder Elbow Surg 2013;22:1623-1627.

11. Kim C-Y, Wiznia DH, Hsiang WR, Pelker RR. The effect of insurance type on patient access to knee arthroplasty and revision under the Affordable Care Act. J Arthroplasty 2015;30:1498-1501.

12. Kenney GM, Zuckerman S, Dubay L, et al. Opting in to the Medicaid Expansion under the ACA: who are the uninsured adults who could gain health insurance coverage? 2012;(August):1-19. http://Www.urban.org/ UploadedPDF/412630-opting-in-medicaid.pdf. Accessed August 15, 2019.

13. Apfeld J, Han A, Stern T, et al. Implications of the Supreme Court ruling on Medicaid. AAOS Now 2012;6: 20-24. 